\section{A silent orchestra: convergent song loss in Hawaiian crickets is repeated, morphologically varied, and widespread}

\author{
Jack G. Rayner, ${ }^{1,3}$ Sarah Aldridge, ${ }^{2}$ Fernando \\ Montealegre-Z, ${ }^{2}$ And Nathan W. Bailey ${ }^{1}$ \\ Manuscript received 29 January 2019; revised 4 February \\ 2019; accepted 20 February 2019. Corresponding Editor: John \\ Pastor. \\ ${ }^{1}$ School of Biology, University of St Andrews, St Andrews \\ KY16 9TH United Kingdom. \\ ${ }^{2}$ School of Life Sciences, University of Lincoln, Lincoln LN6 \\ 7DL United Kingdom. \\ ${ }^{3}$ E-mail: jackgrayner@gmail.com
}

Citation: Rayner, J. G., S. Aldridge, F. Montealegre-Z, and N. W. Bailey. 2019. A silent orchestra: convergent song loss in Hawaiian crickets is repeated, morphologically varied, and widespread. Ecology 00(00):e02694. https://doi.org/10.1002/ecy.2694

Key words: adaptation; convergent evolution; field cricket; host-parasite interaction; natural selection; rapid adaptation; sexual signal; Teleogryllus oceanicus; trait loss.

Host-parasite interactions are predicted to drive the evolution of defenses and counter-defenses, but the ability of either partner to adapt depends on new and advantageous traits arising. The loss of male song in Hawaiian field crickets (Teleogryllus oceanicus) subject to fatal parasitism by eavesdropping flies (Ormia ochracea) is a textbook example of rapid evolution in one such arms race (Dugatkin 2008). Male crickets ordinarily sing to attract females by rubbing their forewings together, which produces sound by exciting acoustic resonating structures formed from modified wing veins (normal-wing, Nw; Fig. 1A). The resulting song is the target of strong sexual selection by conspecific females. However, in Hawaii, male song also attracts female flies that squirt larvae onto males or nearby female crickets; the larvae then burrow into, consume, and ultimately kill the host. The flies thus impose strong natural selection on male song.

Approximately $15 \mathrm{yr}$ ago, Zuk et al. (2006) observed the emergence and rapid spread of silent male mutant T. oceanicus phenotypes in parasitized populations on Kauai and Oahu. Song loss is caused by genetic mutations that greatly reduce or eliminate sound-producing structures by superficially feminizing male wing venation (flat wing, Fw; Fig. 1A): all females have unmodified wings, and are incapable of producing song. Due to its protective effect against the parasitoid fly, the flat wing phenotype spread very rapidly (Zuk et al. 2006). However, flat wing phenotypes are associated with independent genetic architectures on Kauai and Oahu, providing a striking example of convergent evolution on a contemporary timescale (Pascoal et al. 2014). On visits to parasitized cricket populations in 2017 and 2018, we discovered two additional wing phenotypes: small-wing (Sw; Fig. 1B) and curly wing (Cw; Fig. 1B). Small-wing and curly wing differ noticeably from flat wing and from each other, but they all eliminate or severely reduce the acoustic signals that are attractive to flies.

We discovered these reduced-song phenotypes while performing transect surveys in parasitized populations where flat wing morphs have not come to predominate (Fig. 2A). We first identified curly wing in the CC population (initials refer to site codes; Fig. 2A) in 2017, and name it for its similarity with the Drosophila wing mutation described nearly a century ago by Ward (1923). To our knowledge, it has never been described in crickets. In lab populations reared from eggs of $\sim 30$ wild-caught females, curly wing morphology persisted across four generations at similar proportions $(\sim 50 \%)$, strongly suggesting a heritable basis. The trait is observable immediately upon adult eclosion, and other lab populations reared in the same growth chamber do not express it.

Curly wing morphology definitively protects calling males from parasitoid attack relative to typical $\mathrm{Nw}$ males (Fig. 2B,C). First, we found that males with $\mathrm{Nw}$ venation, but exhibiting curly wing morphology like that shown in Fig. 1C, do not sing as loudly as typical $\mathrm{Nw}$ males (Wilcox rank sum test: $\mathrm{N}=15, \quad P<0.001$; Fig. 2B; comparing songs measured in the lab using a CEM DT-805 (CEM, Shenzhen, China) sound level meter $5 \mathrm{~cm}$ from test subjects). Flat wing males also attempt to sing (Schneider et al. 2018), but the amplitude of acoustic stimuli produced during wing movement did not differ between $\mathrm{CwNw}$ and $\mathrm{Fw}$ males $(N=13, P=1.000)$. Like $\mathrm{Fw}$ males, $\mathrm{CwNw}$ males produced variable, but lower-amplitude, peak frequencies (Fig. 2B). Second, we found that the sound reduction caused by curly wing morphology prevents $O$. ochracea attack (Fig. 2C). We performed playback trials at the $\mathrm{CC}$ site using looped calling songs recorded in the lab at $25^{\circ} \pm 1^{\circ} \mathrm{C}$ from $4 \mathrm{Nw}$ and $4 \mathrm{CwNw}$ males. Songs were played on SanDisk MP3 players (SanDisk, Milpitas, California, USA) through Sony SRS-m30 speakers (Sony, Tokyo, Japan) underneath fly traps (modified 1.5- 
a

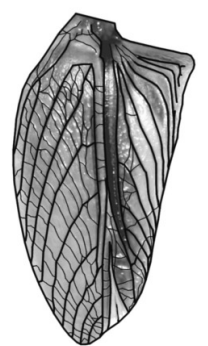

Female

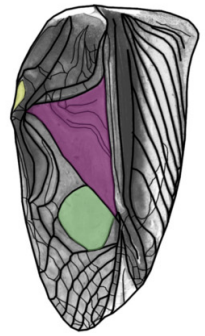

Nw male

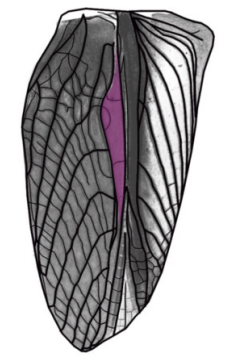

Fw male (Kauai)

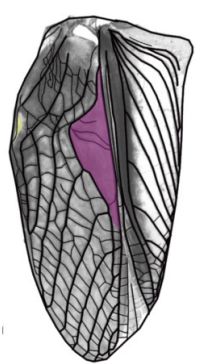

Fw male (Oahu)
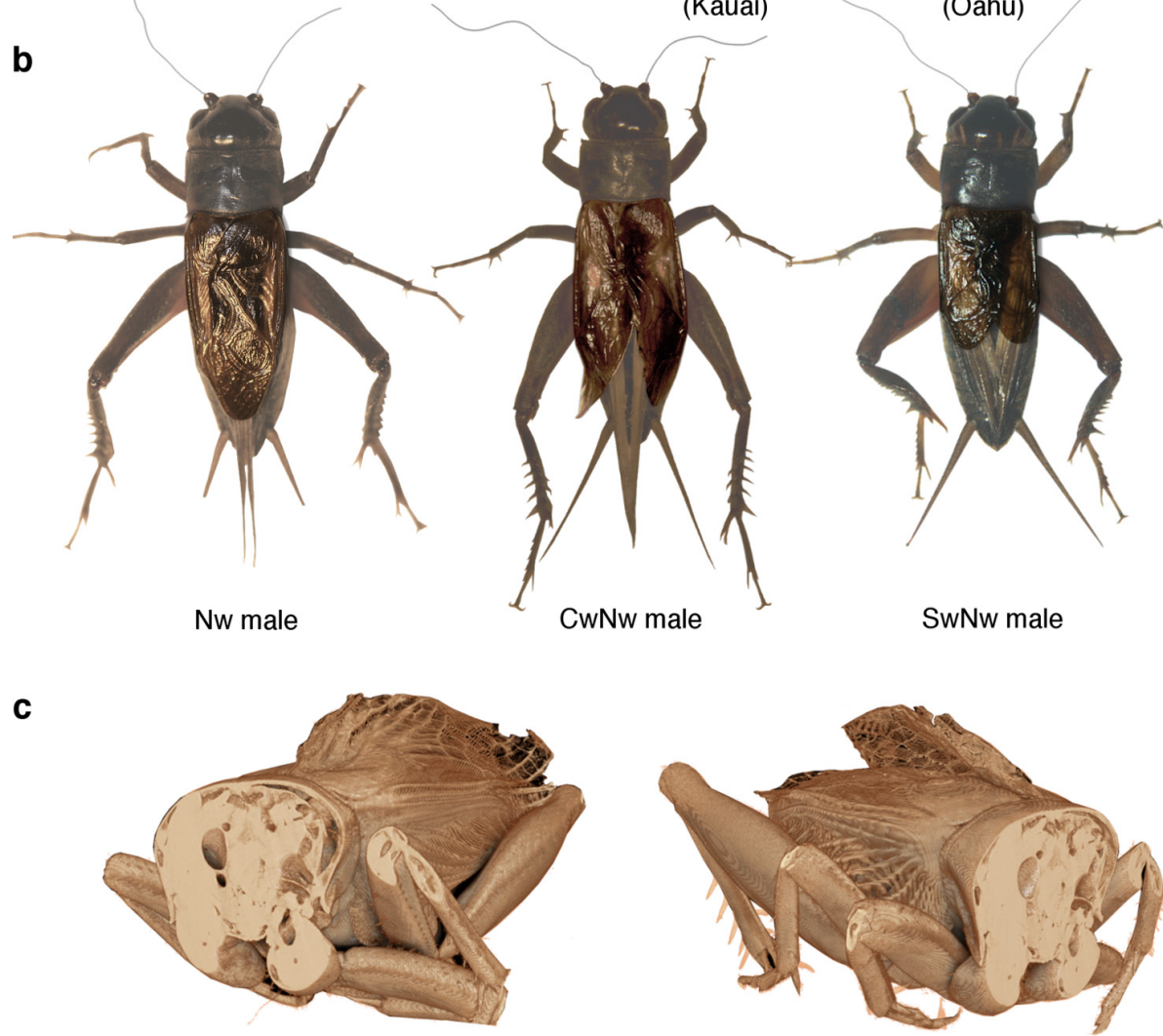

FIG. 1. Alternative male-silencing wing morphs of Hawaiian Teleogryllus oceanicus. (a) Venation variants: traced micrographs showing forewing venation patterns (adapted from Pascoal et al. 2014) of a female and normal-wing (Nw) male and flat-wing (Fw) males from the different islands, with sound-producing structures highlighted (purple, harp; green, mirror; yellow, plectrum). (b) Newly described shape and size variants: typical $\mathrm{Nw}$ male (left) alongside curly wing normal-wing (CwNw) male and smallwing, normal-wing ( $\mathrm{SwNw}$ ) male; note that both forewings and hindwings are reduced. (c) Micro-CT scans of a CwFw male with forewings in resting position and head and thorax omitted, showing how marginal wing surfaces "peel up" and preclude physical engagement during wing movement.

L plastic bottles with the funnel end inverted), broadcast at their originally recorded volumes. Since $\mathrm{Cw}$ males were only found in populations that also contained calling $\mathrm{Nw}$ males (Fig. 2A), we designed playbacks to mimic natural conditions by placing three traps $11 \mathrm{~m}$ apart in a triangle: one typical $\mathrm{Nw}$ song, one $\mathrm{CwNw}$ song, and a third without playback as a negative control. Trials lasted $5 \mathrm{~min}$ and were performed in dry weather between sunset ( 18:10) and 20:30 when the fly is active
(Beckers and Wagner 2012). All pairings of typical Nw and $\mathrm{CwNw}$ song models were repeatedly tested over 4 nights and rotated among speakers between trials. Like the negative controls, $\mathrm{CwNw}$ songs never resulted in a fly entering the trap, whereas typical $\mathrm{Nw}$ songs attracted flies in $28.13 \%$ of trials (paired Wilcoxon signed rank test, $N=64, P<0.001)$.

In the same field season, when surveying a different parasitized population of Hawaiian T. oceanicus (UH in 


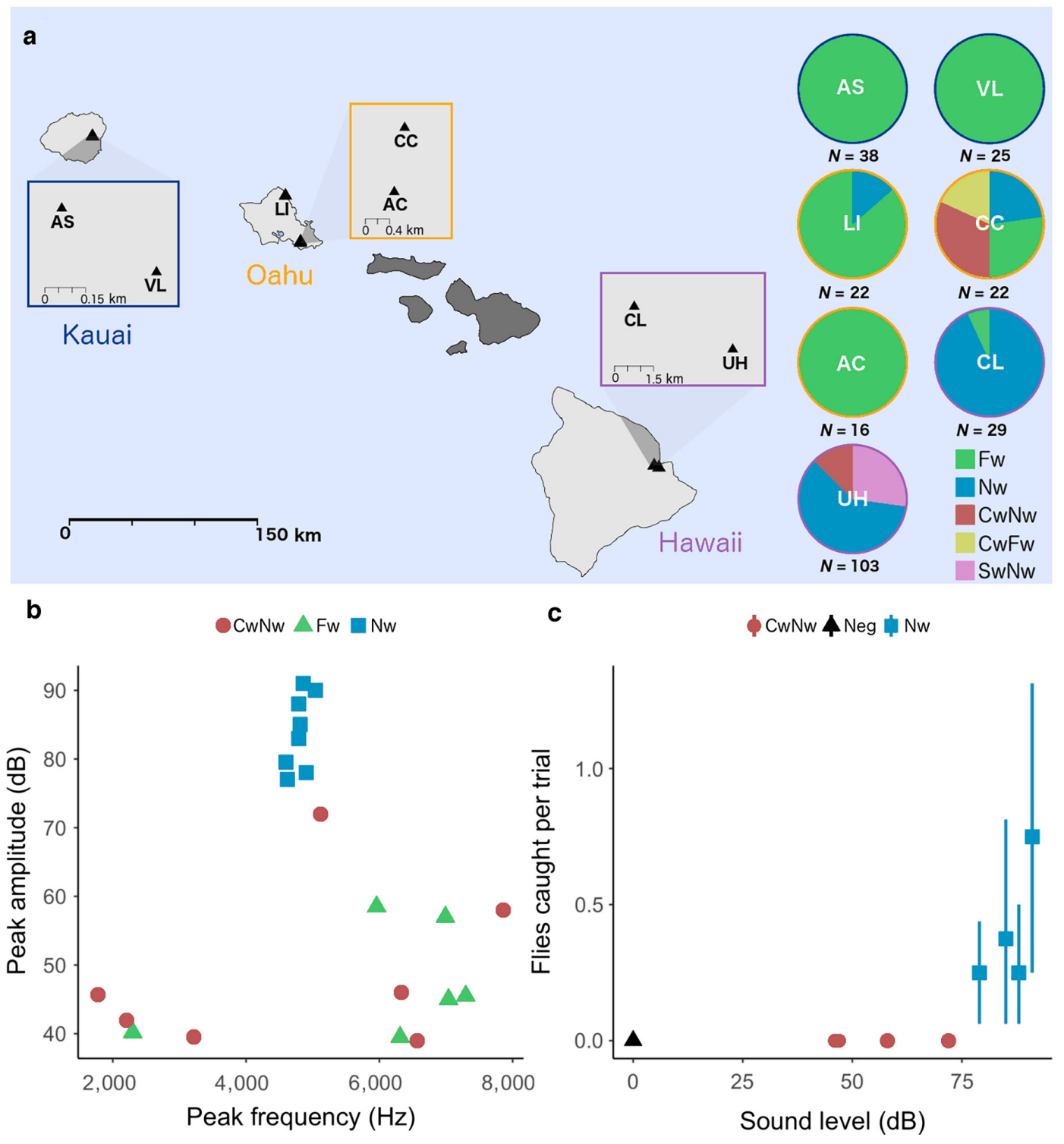

FIG. 2. (a) Distributions of parasitized populations of Teleogryllus oceanicus and proportions of males showing typical Fw and Nw morphology plus newly identified $\mathrm{CwNw}, \mathrm{CwFw}$, and $\mathrm{SwNw}$ phenotypes from 2018 surveys. Two-letter codes correspond to site IDs. (b) Differences in calling song properties for Nw, Fw, and CwNw males recorded using a Sennheiser ME66 microphone (Sennheiser, Wedemark, Germany) $5 \mathrm{~cm}$ from subjects in the lab. (c) Flies attracted to CwNw, negative control and Nw playbacks in the field: points illustrate means and bars are $95 \%$ nonparametric confidence intervals.

Fig. 2A) in which $<5 \%$ of males exhibit flat wing morphology, we noted a substantial proportion of males $(N=28,27.18 \%)$ with unusually small, but normally veined forewings (SwNw, Fig. 1B). We temporarily removed $12 \mathrm{SwNw}$ males from the field and measured courtship song that they produced when exposed to females $(61.83 \pm 2.99 \mathrm{~dB}$ [mean $\pm \mathrm{SE}$ ], see Videos S1-S3). Two of the 12 produced acoustic stimuli below the recordable atmospheric noise level of $\sim 45 \mathrm{~dB}$, so we conservatively dummy-coded these in analyses as producing song at $45 \mathrm{~dB}$. One of the 12 had forewings of differing lengths and sang at up to $80 \mathrm{~dB}$, toward the lower end of the normal range (Balakrishnan and Pollack 1996), but this was the exception. The other 11 produced acoustic signals at substantially lower than normal levels. These observations strongly suggest that their reduced song amplitude also protects $\mathrm{SwNw}$ males against parasitoid attack.

We observed that small-wing morphology not only affected crickets' forewings, which males use to produce song, but also the hindwings, which both sexes use for flight (Fig. 1B). Brachyptery is commonly observed in 
the hindwings only of related species (though not, to our knowledge, in T. oceanicus) and is highly heritable in related species (Roff 1994). An important distinction is that brachypterous forms of field crickets such as Gryllus firmus gain a fitness advantage by divesting energy from maintaining large hindwings and flight muscle, while boosting their attractiveness to females through increased calling effort using the forewings (Crnokrak and Roff 1998). In T. oceanicus, small-wing males are unable to produce ordinary calling or courtship song, owing to their reduced forewings, and so would gain no such benefit.

The initial discovery of flat wing stimulated research into behavioral and physiological consequences of trait loss and rapid evolution, and a population of $T$. oceanicus was recently described on Molokai in which flat wing males produce severely attenuated, broad-band acoustic stimuli (Tinghitella et al. 2018). Our identification of additional protective, reduced-song wing morphs raises many questions. The emergence of alternative adaptive phenotypes may have inhibited the spread of flat wing males and could account for their variable proportions observed in different populations (Zuk et al. 2018). Do flat wing, curly wing and small-wing males differ in attractiveness to females, and does one phenotype have an advantage over others? They all appear capable of co-expression in the same male, and females also express curly wing and small-wing, so it will be important to dissect their genetic architecture.

The recurrent adaptive loss of song across small, fragmented populations of Hawaiian field crickets illustrates the multiple, morphologically varied routes by which this male trait can be functionally lost. Our observations are consistent with recent evidence for high evolvability of trait loss under negative selection (Xie et al. 2018), a phenomenon widely observed among costly sexually selected traits (Wiens 2001), and which may play an important role in rapid adaptation of populations to novel environments or selection pressures. The recurrent disappearance of song in T. oceanicus suggests evolutionary trait or signal loss could be a common means for hosts to evade their parasites, owing to the fitness advantages that arise from evading detection.

\section{ACKNOWLEDGMENTS}

We thank David Forbes and Audrey Grant for help with cricket husbandry. Our manuscript benefitted substantially from comments from the Editor and two anonymous reviewers on an earlier version. This work was supported by funding to N. W. Bailey from the UK Natural Environment Research Council (NE/I027800/1, NE/L011255/1). The micro-CT scanner was funded by the European Research Council, Grant ERCCoG-2017-773067 to F. Montealegre-Z.

\section{Literature Cited}

Balakrishnan, R., and G. S. Pollack. 1996. Recognition of courtship song in the field cricket, Teleogryllus oceanicus. Animal Behaviour 51:353-366.

Beckers, O. M., and W. E. Wagner. 2012. Divergent preferences for song structure between a field cricket and its phonotactic parasitoid. Journal of Insect Behavior 25:467-477.

Crnokrak, P., and D. A. Roff. 1998. The genetic basis of the trade-off between calling and wing morph in males of the cricket Gryllus firmus. Evolution 52:1111-1118.

Dugatkin, L. A. 2008. Principles of animal behavior. Second edition. W.W. Norton and Co., New York, New York, USA.

Pascoal, S., T. Cezard, A. Eik-Nes, K. Gharbi, J. Majewska, E. Payne, M. G. Ritchie, M. Zuk, and N. W. Bailey. 2014. Rapid convergent evolution in wild crickets. Current Biology 24:1369-1374.

Roff, D. A. 1994. Why is there so much genetic variation for wing dimorphism? Researches on Population Ecology 36:145.

Schneider, W. T., C. Rutz, B. Hedwig, and N. W. Bailey. 2018. Vestigial singing behaviour persists after the evolutionary loss of song in crickets. Biology Letters 14:20170654.

Tinghitella, R. M., E. D. Broder, G. A. Gurule-Small, C. J. Hallagan, and J. D. Wilson. 2018. Purring crickets: the evolution of a novel sexual signal. American Naturalist 192:773-782.

Ward, L. 1923. The genetics of curly wing in Drosophila. Another case of balanced lethal factors. Genetics 8:276-300.

Wiens, J. J. 2001. Widespread loss of sexually selected traits: How the peacock lost its spots. Trends in Ecology and Evolution 16:517-521.

Xie, K. T., G. Wang, A. C. Thompson, J. I. Wucherpfennig, T. E. Reimchen, A. D. C. MacColl, D. Schluter, M. A. Bell, K. M. Vasquez, and D. M. Kingsley. 2018. DNA fragility in the parallel evolution of pelvic reduction in stickleback fish. Science 363:81-84.

Zuk, M., N. W. Bailey, B. Gray, and J. T. Rotenberry. 2018. Sexual signal loss: the link between behaviour and rapid evolutionary dynamics in a field cricket. Journal of Animal Ecology 87:623-633.

Zuk, M., J. T. Rotenberry, and R. M. Tinghitella. 2006. Silent night: adaptive disappearance of a sexual signal in a parasitized population of field crickets. Biology Letters 2:521-524.

Additional supporting information may be found in the online version of this article at http://onlinelibrary.wiley.com/doi/10. 1002/ecy.2694/suppinfo 